\title{
Point-to-point practical controller performance in an experimental two-axes belt driven mechanism
}

\author{
Purtoj $^{*}$ and Mulfiyandi \\ Mechanical Engineering Dept., Faculty of Industrial Engineering, Islamic University of Indonesia, Jl. Kaliurang km 14.5 Sleman, \\ Yogyakarta, Indonesia
}

\begin{abstract}
High accuracy, fast response with no or small overshoot, and robust to object uncertainties are such performance required in a point-to-point positioning system. In order to achieve those performances, a good controller is required. A nominal characteristic trajectory following controller is one type of practical controllers which already has shown it good point-to-point positioning performance. Its structure consists of a nominal characteristic trajectory and a compensator. The design of the controller is based on a simple open loop experiment to construct a nominal characteristic trajectory. Information from nominal characteristic trajectory was then used to design the compensator. This practical approach was implemented in several different controlled objects, but limited to, system with a high stiffness mechanism. In this work, a nominal characteristic trajectory following controller was implemented on a low stiffness mechanism. An experimental two-axes belt driven mechanism was used as a controlled object. The actuator to drive the mechanism was electric motors with integrated encoders. Both axes with different characteristics identified through a simple open loop experiments and nominal characteristic trajectory constructed using speed and position data. A proportional integral compensator is selected in this work and was designed according to information from a nominal characteristic trajectory. In order to evaluate controller performance, point to point operation was conducted through simulation and experiment.
\end{abstract}

\section{Introduction}

Point-to-point (PTP) operation is widely used in industrial application. Pick and place operation, NC drill presses and spot welding operation, to name only few example applications of PTP operation. PTP operation as part of positioning systems requires a controller to satisfy such requirements as high accuracy, fast response with no or small overshoot, and robust to object uncertainties. Many kinds of controllers have been proposed and evaluated for positioning systems. The PID controller performance still in investigation dealing with positioning system, in the example, the cascaded PID controller was proposed to reduce nonlinearities factors such as friction and power saturation [1]. The PID controller designed with dominant pole placement method provides one more parameter than the conventional PID controller to get better performances [2]. The sliding mode controller also provides good tracking performance and robustness even in the presence of the parameter uncertainties [3]. Practical controller, nominal characteristic trajectory controller (NCTF) which was one of controller developed to handle point to point operation [4] provides good performance despite its simple design and its procedure. The NCTF controller has a simple structure. It consists of a nominal characteristic trajectory (NCT) and a compensator. Construction of the NCT is simply based on open loop responses of the controlled object. Moreover, the design of the compensator is based on NCT information. Its performance dealing with mechanism with small elasticity effectively compensated vibration cause by mechanical resonance [5]. In this paper, an implementation of nominal characteristic trajectory controller on an experimental two-axes belt driven mechanism which has low stiffness mechanism will be investigated.

\section{Introduction}

The structure of the NCTF control system is shown in Fig. 1. The controller consists of a nominal characteristic trajectory (NCT) and a compensator. The controller output is signal $u_{p}$, this signal is used to drive the object. The controller inputs are error, $e$, and object motion, \&. In principle, the controller compares the object motion input, $\&$, with the error-rate provided by predetermined NCT, \& at a certain error. The difference between the actual error-rate of the object motion and that of the NCT is signal $u_{p}$, which is the output of the NCT. If the object motion perfectly follows the NCT, the value of signal $u_{p}$ is zero. Thus, no action is performed by the compensator. When the signal $u_{p}$ is not zero, the compensator forces the object motion so that the value of signal $u_{p}$ is zero. The objective of the compensator is to make the object motion

\footnotetext{
*Corresponding author: purtojo@uii.ac.id
} 
follow the NCT, and finishes at the origin of the phaseplane $(e=0, \&=0)$.

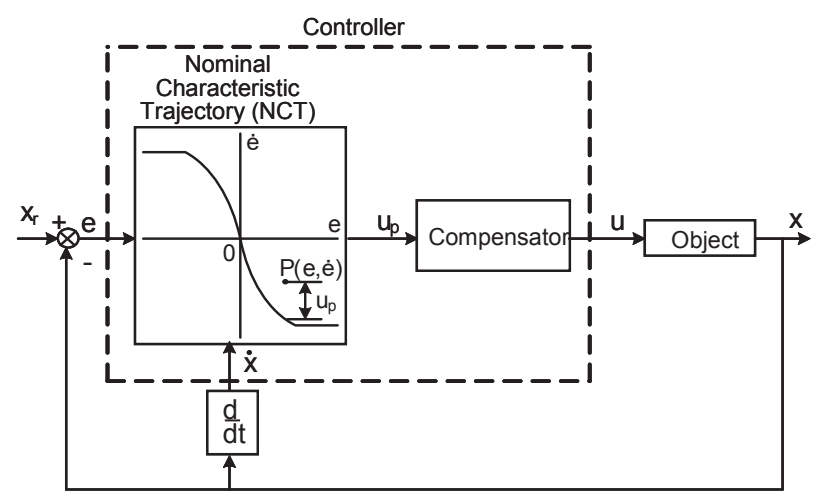

Fig 1. Structure of the NCTF control system

In order to determine the $\mathrm{NCT}$, a simple open-loop experiment has to be conducted. In the experiment, stepwise inputs are applied to the object, and displacement and velocity responses of the object are measured. Fig. 2 (a) shows the stepwise input, and the resulting velocity and displacement responses, normalized respective to its input heights. The rated input to the actuator $u_{r}$ is used as a value of the stepwise inputs.

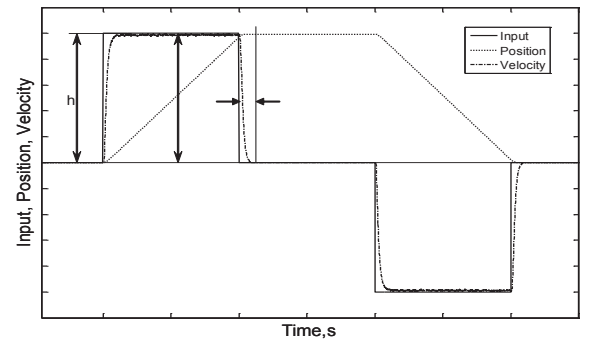

(a)

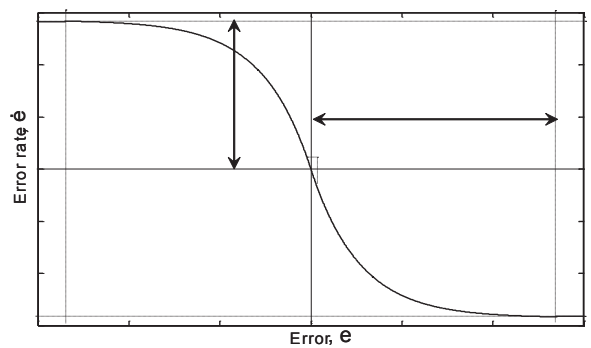

(b)

Fig 2. (a) Open-loop responses (b) NCT

The velocity and displacement responses are used to determine the NCT. The phase plane of the NCT has a horizontal axis of error, $e$, and a vertical axis of error-rate, $\dot{e}$, as shown in Fig. 2 (b). The horizontal axis shows the error from the desired position. Therefore, the values are taken from the displacement data of the open-loop responses. The error-rate is the velocity data within the deceleration process. In Fig. 2 (a), $\mathrm{h}$ is the maximum velocity, which depends on the input step height and gradually decreasing to zero. Thus the pair data of error and error-rate construct the NCT.

\section{Experimental Setup}

An experimental two-axes belt driven mechanism as a controlled object is shown in Fig. 3. It represents an industrial $\mathrm{CNC}$ machine. The mechanism provides three axes perpendicular motion, however discussion is limited to $\mathrm{X}$ and $\mathrm{Y}$ direction only. The table mounted on linear bearing slides along a circular rod. DC motors with integrated rotary encoders are used as actuators. Those are received signal from motor drivers connected to an Arduino Mega 256. The control signal is sent from Scilab $\mathrm{Xcos}$ model build in a personal computer.

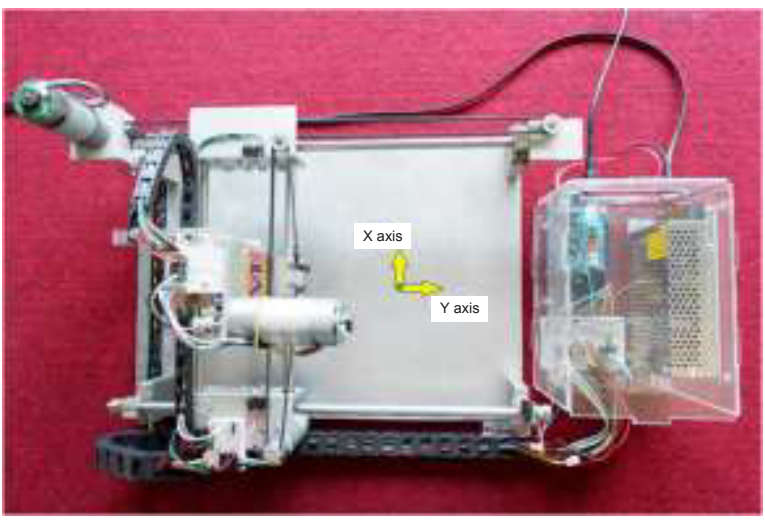

Fig 3. Experimental two-axes belt driven positioning system

The open-loop experiment using stepwise input had been conducted in both axes of the mechanism. Maximum input voltage was $u_{r}=12$ volts. Fig. 4 shows the velocity and displacement responses on both-axes.

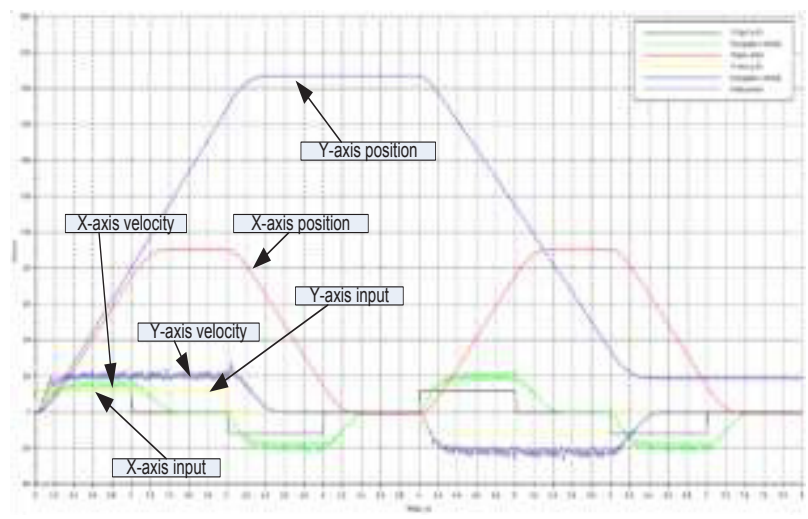

Fig 4. System responses to a stepwise inputs

\subsection{NCT}

Velocity and position data of system responses are then used to construct the NCT of both axes. The NCT of $\mathrm{x}$ axis is shown Figure 5 (a) and Figure 5 (b) shows the NCT of Y-axis. Two important parameters in the NCT are the maximum error rate indicated by $\mathrm{h}$ and the near-zero slope of the NCT indicated by $\mathrm{m}$. These parameters are required to design the compensator. $\mathrm{X}$-axis NCT parameters are $\mathrm{hx}=25.31 \mathrm{~mm} / \mathrm{s}$ and $\mathrm{mx}=-124.5$, and $\mathrm{Y}$-axis NCT parameters are hy=25.71 mm/s and $m y=-25.06$. 


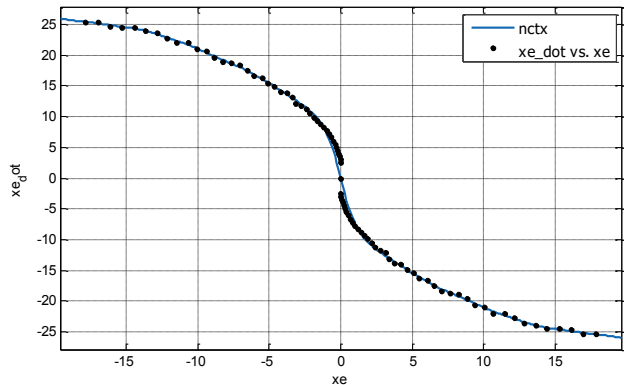

(a)

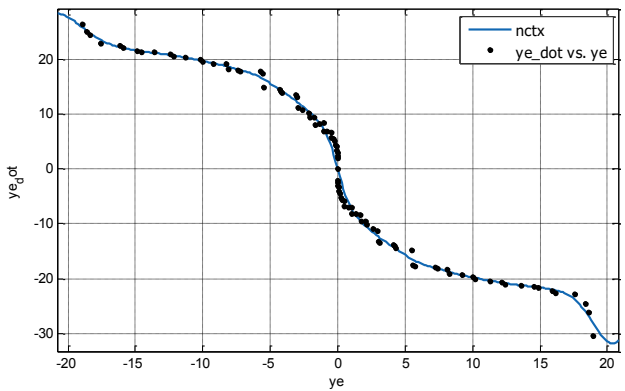

(b)

Fig 5. (a) NCT of X-axis, and (b) NCT of Y-axis

\subsection{Compensator Design}

In this study PI controller is used as a compensator to control the movement of the controlled object.

$$
G_{C}(s)=K_{P}+\frac{K_{i}}{s}
$$

PI compensator can be expressed as a function of the natural frequency and damping ratio as follows (Wahyudi, Sato, \& Shimokohbe, 2005):

$$
\begin{gathered}
K_{p}=\frac{2 \zeta \omega_{n}}{\alpha K}=\frac{2 \zeta \omega_{n} u_{r}}{m h} \\
K_{i}=\frac{\omega_{n}^{2}}{\alpha K}=\frac{\omega_{n}^{2} u_{r}}{m h}
\end{gathered}
$$

\section{Positioning Performances}

Performance of the designed NCTF controller evaluated through simulation and experiment. The evaluation was conducted by driving the controlled object to a several inputs, $10 \mathrm{~mm}$ to $50 \mathrm{~mm}$ input. Figure 6 shows positioning performance of NCTF controller to a $10 \mathrm{~mm}$ input, Figure 6 (a) for $\mathrm{X}$-axis and Figure 6 (b) for Y-axis respectively. In this paper only $10 \mathrm{~mm}$ input presented since the other responses to different input is similar.

Designed NCTF controller had been evaluated for position control using the PI controller as a compensator. This investigation shows that the maximum steady state error achieved on the $\mathrm{X}$ axis is $8.73 \%$ for a $10 \mathrm{~mm}$ input, and lower to higher inputs. Steady state condition is achieved with settling time of 0.28 seconds. On the $\mathrm{Y}$ axis the maximum steady state errors also present for a $10 \mathrm{~mm}$ input, which is $8.56 \%$, and lower to higher inputs. The settling time is 0.2 seconds. Since the input larger $20 \mathrm{~mm}$ is out of NCT, the settling time obviously is slower, however the oscillation is reduced. Thus, because the system has a constant linear velocity, so the further the set point value is given, then the longer the system reaches the desired value.

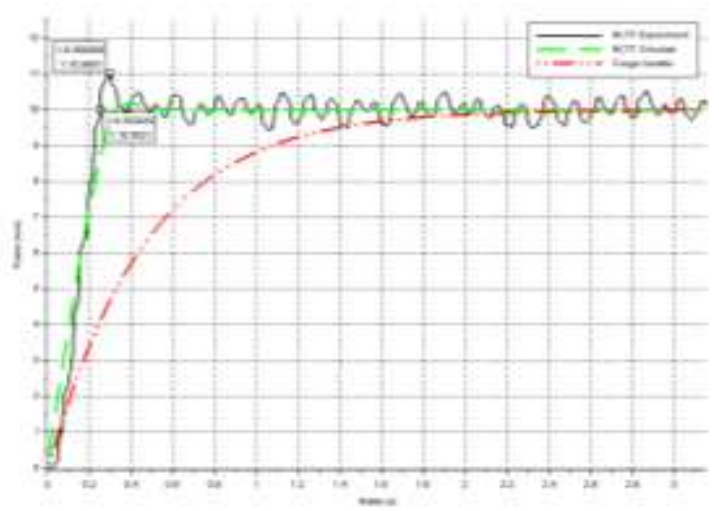

(a)

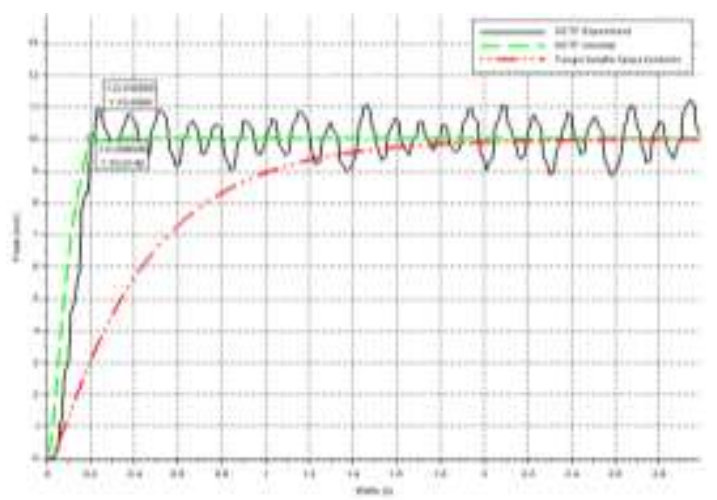

(b)

Fig 5. Positioning performance to a $10 \mathrm{~mm}$ input (a) $\mathrm{X}$-axis, and (b) Y-axis.

Oscillation about desired position was occurring on both axes. The source of oscillation was the hardware selection problem, which is the belt to drive the mechanism. The stiffness of the belt is very low and not sufficient to withstand the inertia of the system. As shown in the $\mathrm{X}$-axis, the oscillation is smaller compared to oscillation in $\mathrm{Y}$-axis. This is related to the mass of components moved along each axis. Since the belt has a high elasticity compared to other more precise drive transmissions such as ball screw, it cannot withstand the inertia of the moving components. Overall the position control system has been designed to run in accordance with the expectation. The system response drive by the NCTF controller was able to accelerate the rise time on both the $\mathrm{X}$-axis and the $\mathrm{Y}$-axis. Although the system has a sufficiently large steady state error value, but it's still within the allowed tolerance of $2-10 \%$ of the steady state error. 


\section{Positioning Performances}

The NCTF controller design procedure had been successfully applied to an experimental two-axes positioning system with belt driven mechanism. The open loop experiment provided necessary information to construct NCT and consecutively to design the PI compensators. Controller design is simple and successfully implemented to the controlled object. Performance evaluation through simulation and experiment show that the designed NCTF controller able to accelerate the rise time. However, controller positioning performance deteriorated by the very low stiffness of the belt. Oscillation about the steady state position occurred constantly. Thus, selection of sufficient hardware properties is a must prior to implementation of the controller.

\section{References}

1. K.H. Ng, C.F. Yeong, E.L.M. Su, L.X. Wong. Procedia Engineering 41, 244-250 (2012).

2. P.D. Mandić, T.B. Šekara, M.P. Lazarević, M. Bošković. ISA Transactions 67, 76-86 (2017).

3. M.C. Pai. J of the Franklin Institute 347(10), 1837 1849 (2010).

4. Wahyudi, K. Sato, A. Shimokohbe. Precision E. 27(2), 157-169 (2003)

5. M.F.M. Yakub, W. Martono, R. Akmeliawati. The IEEE Control \& System Graduate Research Colloquium pp. 61-67 (2010).

6. Wahyudi, K. Sato, A. Shimokohbe. Robotics and Autonomous Systems 52(2-3), 247-256 (2005). 